\title{
Expression of Toll-like Receptors, Pro-, and Anti- inflammatory Cytokines in Relation to Gut Microbiota in Irritable Bowel Syndrome: The Evidence for Its Micro-organic Basis
}

\author{
Ratnakar Shukla, ${ }^{1}$ Ujjala Ghoshal, ${ }^{1 *}$ Prabhat Ranjan, ${ }^{1}$ and Uday C Ghoshal ${ }^{2}$ \\ Departments of ${ }^{I}$ Microbiology and ${ }^{2}$ Gastroenterology, Sanjay Gandhi Postgraduate Institute of Medical Sciences, Lucknow, India
}

\section{Background/Aims}

A Subset of patients with irritable bowel syndrome (IBS) may have mild inflammation due to immune activation. Toll-like receptors (TLRs) and cytokines may cause intestinal inflammation. We studied their expression in relation to gut microbiota.

\section{Methods}

Expression of TLRs and cytokines was assessed in 47 IBS patients (Rome III) and 25 controls using quantitative real-time polymerase chain reaction. Immunohistochemistry was further performed to confirm the expression of TLR- 4 and TLR-5.

\section{Results}

Of 47 patients with IBS, 20 had constipation (IBS-C), 20 diarrhea (IBS-D), and 7 unclassified (IBS-U). The mRNA levels of TLR-4 and TLR-5 were up-regulated in IBS patients than controls $(P=0.013$ and $P<0.001$, respectively). Expression of TLR-4 and TLR-5 at protein level was 4.2-folds and 6.6-folds higher in IBS-D than controls. The mRNA levels of IL-6 $(P=0.003), C-X-C$ motif chemokine ligand $11(C X C L-11)(P<0.001)$ and $C-X-C$ motif chemokine receptor $3(C X C R-3)(P<0.001)$ were higher among IBS patients than controls. Expression of IL-6 $(P=0.002)$, CXCL-11 $(P<0.001)$, and CXCR-3 $(P<0.001)$ were up-regulated and IL-10 $(P=0.012)$ was down-regulated in IBS-D patients than controls. Positive correlation was seen between TLR-4 and IL-6 $(P=0.043)$, CXCR-3, and CXCL$11(P=0.047)$, and IL-6 and CXCR-3 $(P=0.003)$. Stool frequency per week showed positive correlation with mRNA levels of TLR-4 $(P=0.016)$ and CXCR-3 $(P=0.005)$, but inversely correlated with IL-10 $(P=0.002)$. Copy number of Lactobacillus $(P=0.045)$ and Bifidobacterium ( $P=0.011)$ showed correlation with IL-10 in IBS-C, while Gram-positive $(P=0.031)$ and Gram-negative bacteria $(P$ $=0.010$ ) showed correlation with CXCL-11 in IBS-D patients.

\section{Conclusions}

Altered immune activation in response to dysbiotic microbiota may promote intestinal inflammation in a subset of patients with IBS.

(J Neurogastroenterol Motil 2018;24:628-642)

Key Words

Cytokines; Immunohistochemistry; Lipopolysaccharide; Peptidoglycan; Toll-like receptors

Received: July 23, 2018 Revised: None Accepted: August 24, 2018

(c) This is an Open Access article distributed under the terms of the Creative Commons Attribution Non-Commercial License (http://creativecommons. org/licenses/by-nc/4.0) which permits unrestricted non-commercial use, distribution, and reproduction in any medium, provided the original work is properly cited.

*Correspondence: Ujjala Ghoshal, MD

Department of Microbiology, Sanjay Gandhi Postgraduate Institute of Medical Sciences, Lucknow 226014, India Tel: +91-522-2495221, Fax: +91-522-2668129, E-mail: ujjalaghoshal@yahoo.co.in 


\section{Introduction}

Irritable bowel syndrome (IBS) is a common non-organic bowel disorder, which is associated with chronic abdominal pain, bloating, discomfort, and irregular bowel movement. ${ }^{1}$ The pathophysiology of IBS is poorly understood; however, several possible mechanisms have been proposed including altered gastrointestinal (GI) microbiota, infectious gastroenteritis, visceral hypersensitivity, dysregulation of gut-brain axis, chronic stress, and genetic factors. ${ }^{1-3}$ Recent studies reported that mild inflammation exists at least in a subgroup of patients with IBS, which is associated with gut dysfunction, increased permeability of intestinal epithelial cells (IECs), and altered profile of proinflammatory and anti-inflammatory cytokines. ${ }^{1,4}$ However, the underlying pathophysiology of low-grade inflammation among patients with IBS remains unknown.

The low-grade inflammation among patients with IBS might be due to aberrant stimulation of innate and adaptive immune responses. ${ }^{5,6}$ Dysregulated mucosal and systemic immune responses have been shown among patients with IBS. $^{7}$ Pattern-recognition receptors, like toll-like receptors (TLRs), are important components of the innate immune system and play a central role in mucosal immune response among patients with IBS. $^{8}$ TLRs recognize conserved pathogen-associated molecular patterns allowing the initiation of innate immune response via triggering the expression of cytokines and chemokines. ${ }^{4}$

Almost all TLRs are expressed on the intestinal epithelial cells in the small intestine as well as in the colon. ${ }^{9}$ The interaction between TLRs and gut microbiota contributes to the maintenance of the integrity of the intestinal epithelial barrier and homeostasis. ${ }^{10,11}$ However, qualitative or quantitative alterations in gut microbiota play a pivotal role in perpetuating the altered host immune response. One study from our center showed that Gram-negative and segmented filamentous bacteria were higher, while Gram-positive bacteria were comparable among patients with IBS than controls. ${ }^{3}$ TLRs recognize a wide range of microbial ligands such as polypeptides of Gram-positive bacteria (TLR-2), lipopolysaccharides of Gram-negative bacteria (TLR-4) and flagellum proteins of filamentous bacteria (TLR-5). ${ }^{8}$ Binding of these ligands to receptors results in activation of a downstream cascade of cytokines and chemokines production leading to intestinal inflammation. ${ }^{12}$

In addition to innate immunity, TLRs also help in the activation of adaptive immune response. Cross talk between gut microbiota and IECs induces the differentiation of T helper (Th) cells into Th1 and Th2 cells. ${ }^{13}$ Previous studies reported that TLR-2 was associated with Th2 cytokines production and TLR-4 with Th1 cytokine production. ${ }^{14,15}$ Recently, a study showed that TLR-2 ligands induce the production of IL-10, while TLR-4 ligands induce the production of proinflammatory cytokine IL-12 (p70) and reduce IL-10 in the dendritic cells. ${ }^{14}$ Published data indicate that stimulation of TLR-5 with flagellin protein induces the expression of chemokine C-X-C motif chemokine ligand 11 (CXCL-11), which plays an important role in intestinal inflammation. ${ }^{16}$ The receptor of CXCL-11 is expressed by Th1 cells (C-X-C motif chemokine receptor 3 [CXCR-3]); binding of CXCL-11 with CXCR-3 receptor stimulates the Th1 cells to release the proinflammatory cytokines like IL-6. ${ }^{16,17}$ Recently, one study has shown that IL-6 is an important mediator of gut dysfunction among patients with inflammatory bowel disease (IBD). ${ }^{17,18}$ Moreover, CXCR-3 inhibition reduces the expression of IL-6 among patients with IBD. ${ }^{17}$ To the best of our knowledge, mRNA expression of CXCL-11 and its receptor CXCR-3 has not been studied among patients with IBS so far. Thus, based on the above evidence, we hypothesized that TLR mediated cytokines and chemokines in relation to altered gut microbiota or pathogen-derived products may be involved in pathogenesis of intestinal inflammation. Scanty data have been reported on this issue and most of the studies are performed in peripheral blood. ${ }^{12,19}$ Therefore, this study was designed with the following objectives; to study (1) expression of TLR-2, 4, and 5 in colonic biopsies among patients with IBS and controls, (2) expression of proinflammatory (IL-6, CXCL-11, and CXCR-3) and anti-inflammatory cytokines (IL-10) in colonic biopsies among patients with IBS and controls, and (3) relationship between mRNA levels of TLRs, cytokines, and chemokines with the altered fecal microbiota published previously from our center. ${ }^{3}$

\section{Materials and Methods}

\section{Study Subjects}

Patients with IBS attending the Gastroenterology out-patient clinic of a super specialty multi-level university hospital from northern India and fulfilling the Rome III criteria were recruited. Patients with IBS were classified into 3 subtypes viz diarrheapredominant (IBS-D), constipation-predominant (IBS-C), and un-subtyped (IBS-U) using Rome III criteria. Subjects without any functional GI disorder by Rome III criteria undergoing flexible sigmoidoscopy for per rectal bleeding that did not reveal any abnormality (except piles) were included as controls. Patients and controls subjects were excluded if they had organic or metabolic 
diseases or those who received antibiotics, probiotics, prokinetics, or antispasmodic drugs within the previous 4 weeks. Each patient was subjected to clinical investigations such as stool microscopy and occult blood testing, hematology, thyroid function test, and proctosigmoidoscopy. Written informed consent was obtained from all the participants. The study protocol was approved by the Institutional Ethical Committee (No. 2012-132-EMP-64).

\section{Assessment of Clinical Symptoms}

Patients with IBS and controls were interviewed using a locally translated and validated Rome III diagnostic questionnaire ${ }^{20}$ to record demographic and clinical symptoms of IBS such as abdominal pain or discomfort, bloating, straining, the passage of mucus, altered bowel habits, and type of stool (using Bristol stool chart with pictorial presentation and descriptors).

\section{Collection and Processing of Biopsy Tissues}

Patients with IBS and controls were subjected to proctosigmoidoscopy. During a sigmoidoscopy, 3 biopsies from the sigmoid colon were collected in Trizol solution (Invitrogen, Carlsbad, CA, USA). All biopsy samples were snap frozen in liquid nitrogen and stored at $-80^{\circ} \mathrm{C}$ for RNA extraction. Additionally, 3 biopsies were collected in $10 \%$ formalin for immunohistochemistry.

\section{Quantitative Real-time Polymerase Chain Reaction}

Total RNA from colonic tissues was extracted using Qiagen RNeasy kit (Qiagen Inc, Valencia, CA, USA) according to the manufacturer's protocol. Quality and quantity of RNA were confirmed by NanoDrop ND-2000 Spectrophotometer (NanoDrop products, Wilmington, DE, USA). RNA (100 ng) was reverse transcribed into cDNA using cDNA synthesis kit (High-Capacity cDNA Reverse Transcription Kits; Applied Biosystems, Carlsbad, CA, USA), according to manufacturer's instructions. Quantitative real-time polymerase chain reaction (qRT-PCR) was performed in a reaction volume of $25 \mu \mathrm{L}$, containing $12.5 \mu \mathrm{L}$ of SYBR Green master mixture (Applied Biosystems, Carlsbad, USA), $0.25 \mu \mathrm{L}$ of $10 \mathrm{pmol}$ of forward and reverse primers and $2 \mu \mathrm{L}$ of $\mathrm{cDNA}$. All reactions were run in duplicate in Corbett Research 6000 Q-PCR instrument (Rotor gene 6000 software, Sydney, Australia). Primer sequences of target genes are listed in Table $1 .^{21-27}$ Beta-actin was used as a housekeeping gene to normalize the reaction volume of RNA. Thermal cycling conditions for target genes were as follows: initially samples were heated at $95^{\circ} \mathrm{C}$ for 10 minutes to activate AmpliTaq Gold DNA polymerase followed by 40 cycles of amplification via denaturation at $95^{\circ} \mathrm{C}$ for 15 seconds, annealing at optimal temperature (Table 2) for 30 seconds, extension at $72^{\circ} \mathrm{C}$ for 30 seconds. Melting curve analysis was also run with increasing temperature from $60^{\circ} \mathrm{C}$ to $95^{\circ} \mathrm{C}$ (at a regular increment of $0.5^{\circ} \mathrm{C}$ for 5 seconds) to assure that fluorescence is coming from our desired amplicon and not from primer dimer or non-specific PCR amplified products. In addition, quality of qRT-PCR products was confirmed by subjecting to $2 \%$ agarose gel electrophoresis. Replacing template RNA with diethyl pyrocarbonate-water (Sigma, St. Louis, MO, USA) was used as negative control for qRT-PCR. Data were normalized with housekeeping $\beta$-actin gene and relative fold change for target gene was presented using $2^{-\Delta \mathrm{Ct}}$ comparative $\mathrm{Ct}$ method.

\section{Immunohistochemistry}

Colonic biopsies were immediately fixed in formalin and embedded in paraffin as per standard protocol. ${ }^{28}$ Three to five micra sections were cut using microtome (Reichert-Jung 2030 Biocut; Leica, Heidelberg, Germany) and mounted onto clean poly Llysine coated glass slides. The slides were dried for 4-6 hours at $60^{\circ} \mathrm{C}$, de-paraffinized in xylene and rehydrated using decreasing grades of alcohol series. Sodium citrate buffer (1.5 M, pH 6.0) was used for antigen retrieval. Slides were kept in a microoven at $95^{\circ} \mathrm{C}$ for 15 minutes each for 2 times and left to cool in the antigen retrieval buffer. Slides were washed 3 times with phosphate-buffered saline (PBS; pH 7.4). Sections were incubated with $3 \%$ hydrogen peroxide in methanol and kept in dark for 30 minutes to block the endogenous peroxidase activity. The sections were washed 3 times with PBS. Non-specific antigens were blocked with $5 \%$ sheep serum at room temperature for 1 hour. Thereafter, sections were incubated with the mouse monoclonal anti-TLR-4 antibody (1:100 dilution; Abcam, Milton, Cambridge, UK) and rabbit polyclonal anti-TLR-5 (1:50 dilution; Abcam, Cambridge, UK) at room temperature for 1 hour in a humidified chamber. The primary antibody was replaced with PBS for negative controls. Slides were washed 3 times with PBS. Subsequently, sections were incubated with the biotinylated secondary antibody (Novacastra; Leica Biosystems Newcastle Ltd, Newcastle Upon Tyne, UK) for 45 minutes at room temperature. After that, sections were incubated with 3, 3'-diaminobenzidine (DAKO, Glostrup, Denmark) to visualize the reaction products and counterstained with hematoxylin. The sections were washed with water, dehydrated with several grades of alcohols (30\%, $50 \%, 70 \%$, and $100 \%$ ) and xylene. Finally, sections were mounted with mixture of distyrene, plasticizer, and xylene (DPX) mountant. Three images for each slide having consistent staining were taken using Nikon Eclipse Ci H600L (Nikon, Tokyo, Japan) microscope at $10 \times$ magnification. 
Table 1. Primer Sequences for Messenger RNA Expression of Target Genes

\begin{tabular}{|c|c|c|c|c|}
\hline Target gene & Primer sequence (5'-3') & Product size (bp) & Annealing temp $\left({ }^{\circ} \mathrm{C}\right)$ & Ref \\
\hline \multirow[t]{2}{*}{ TLR-2 } & FP: CTGCAAGCTGCGGAAGATAAT & 107 & 56 & 21 \\
\hline & RP: AGGACTTTATCGCAGCTCTCAGA & & & \\
\hline \multirow[t]{2}{*}{ TLR-4 } & FP: TGCAATGGATCAAGGACCAGAGGC & 449 & 68 & 22 \\
\hline & RP: GTGCTGGGACACCACAACAATCACC & & & \\
\hline \multirow[t]{2}{*}{ TLR-5 } & FP: GCATTAAGGGGACTAAGCCTC & 350 & 58.6 & 23 \\
\hline & RP: AAAAGGGAGAACTTTAGGGACT & & & \\
\hline \multirow[t]{2}{*}{ IL-6 } & FP: GTGAAAGCAGCAAAGAGGCA & 276 & 58 & 24 \\
\hline & RP:TTGGGTCAGGGGTGGTTATT & & & \\
\hline \multirow[t]{2}{*}{ IL-10 } & FP: AGTCGCCACCCTGATGTCTC & 223 & 58.2 & 25 \\
\hline & RP: CCTGGGGGAGAACCTGAAG & & & \\
\hline \multirow[t]{2}{*}{ CXCL-11 } & FP: CCTTGGCTGTGATATTGTGTGC & 101 & 62.7 & 26 \\
\hline & RP: CACTTTCACTGCTTTTACCCCAG & & & \\
\hline \multirow[t]{2}{*}{ CXCR-3 } & FP: AGGTGCCCTCTTCAACATCAA & 101 & 63.7 & 26 \\
\hline & RP: GGTAGAGCTGGGTGGCATGA & & & \\
\hline \multirow[t]{2}{*}{$\beta$-actin } & FP: CATGCCATCCTGCGTCTGGACC & 400 & 58 & 27 \\
\hline & RP:ACATGGTGGTGCCGCCAGACA & & & \\
\hline
\end{tabular}

bp, base pair; TLR, toll-like receptor; CXCL-11, C-X-C motif chemokine ligand 11; CXCR-3, C-X-C motif chemokine receptor 3.

Table 2. Demographic and Clinical Symptoms of Patients With Irritable Bowel Syndrome and Controls

\begin{tabular}{|c|c|c|c|}
\hline Parameters & $\operatorname{IBS}(\mathrm{n}=47)$ & Controls $(n=25)$ & $P$-value \\
\hline Age (median [range], yr) & $34(19-68)$ & $40(20-68)$ & $0.271^{\mathrm{a}}$ \\
\hline Gender (male $[\%]$ ) & $39(83 \%)$ & $22(88 \%)$ & 0.573 \\
\hline \multicolumn{4}{|l|}{ Predominant bowel habits (Rome III criteria) } \\
\hline IBS-C & $20(43 \%)$ & - & - \\
\hline IBS-D & $20(43 \%)$ & - & - \\
\hline IBS-U & $7(14 \%)$ & - & - \\
\hline \multicolumn{4}{|l|}{ Clinical Symptoms } \\
\hline Visible abdominal distension & $34(72 \%)$ & 0 & $<0.001$ \\
\hline Abdominal bloating/feeling of abdominal distension $>1 / 4$ of days & $22(47 \%)$ & 0 & $<0.001$ \\
\hline Abdominal pain & $35(74 \%)$ & 0 & $<0.001$ \\
\hline Abdominal discomfort & $47(100 \%)$ & 0 & $<0.001$ \\
\hline Relief of pain/discomfort with bowel movement & $46(98 \%)$ & 0 & $<0.001$ \\
\hline More frequent stool at onset of pain & $13(28 \%)$ & 0 & 0.004 \\
\hline Loose stool at onset of pain & $14(30 \%)$ & 0 & 0.002 \\
\hline Urgency & $17(36 \%)$ & 0 & 0.001 \\
\hline Passage of mucus & $35(74 \%)$ & 0 & $<0.001$ \\
\hline Passage of mucus $>1 / 4$ of defecation & $14(28 \%)$ & 0 & 0.002 \\
\hline Feeling of incomplete evacuation & $45(96 \%)$ & $7(28 \%)$ & $<0.001$ \\
\hline Feeling of incomplete evacuation $>1 / 4$ of defecation & $29(62 \%)$ & 0 & $<0.001$ \\
\hline Straining during defecation & $27(57 \%)$ & 0 & $<0.001$ \\
\hline Irregular stool form & $33(70 \%)$ & $7(28 \%)$ & 0.001 \\
\hline Irregular stool frequency & $39(83 \%)$ & $9(36 \%)$ & $<0.001$ \\
\hline
\end{tabular}

${ }^{\mathrm{a}}$ Non-parametric Mann-Whitney $U$ test. Chi-square test was used for all the other categorical data.

IBS, irritable bowel syndrome; IBS-C, constipation-predominant IBS; IBS-D, diarrhea-predominant IBS; IBS-U, unsubtyped IBS; $P<0.05$ was significant. 


\section{Analysis of Gut Microbiota}

Analysis of fecal microbiota was performed in the same group of IBS patients as described previously. ${ }^{3}$ Fecal DNA extraction and real-time PCR for amplification of $16 \mathrm{~S}$ rRNA gene of target bacteria were reported previously. ${ }^{3}$ In this study, we examined the correlation between the copy number of target bacteria with mRNA level of TLRs, cytokines, and chemokines among patients with IBS.

\section{Statistical Methods}

The mRNA levels of TLRs, cytokines, and chemokines are presented as median and range. Chi-square and Mann-Whitney $U$ tests were used for categorical and continuous data, respectively. The comparative mean Ct method $\left(2^{-\Delta \mathrm{Ct}}\right)$ is used to express the fold change of mRNA level of target gene. Mann-Whitney $U$ test was used to compare the mRNA expression of target gene between patients with IBS and controls. The non-parametric Kruskal-Wallis $\mathrm{H}$ test with post hoc Bonferroni test was applied for comparison of more than 2 groups. Spearman correlation was used to determine the relationship between mRNA level of TLRs, cytokines, and chemokines with clinical symptoms and copy number of target bacteria. ImageJ software version 1.46r (Wayne Rasband, National Institutes of Health, Bethesda, MD, USA; http://rsb.info.nih.gov/ ij/http://rsb.info.nih.gov/ij/) was used for quantitative analysis of IHC image for TLR-4 and TLR-5. ${ }^{29}$ An average value of three fields for each image was considered as percentage staining of positive cells. $P$-values less than 0.05 were considered as significant. All data were analyzed with Graph Prism version 5.0 (GraphPad Software Inc, La Jolla, CA, USA) and SPSS version 15.0 (IBM Corp, Armonk, NY, USA).

\section{Results}

\section{Demographic and Clinical Characteristics of Patients With Irritable Bowel Syndrome and Controls}

A total of 47 patients with IBS and 25 controls comparable in age (median 34 years [19-68] vs 40 [20-68], $P=0.271$ ) and gen$\operatorname{der}(39 / 47$ [83\%] vs 22/25 [88\%] male, $P=0.573)$ were included. Patients with IBS were sub-typed into IBS-D ( $\mathrm{n}=20)$, IBS-C ( $n=20)$, and IBS-U ( $n=7)$ using the Rome III criteria. Clinical characteristics of the patients with IBS and controls are presented in Table 2.

\section{Expression of Messenger RNA Level of Toll-like Receptors Among Patients With Irritable Bowel Syndrome and Controls}

There was no difference in mRNA level of TLR-2 among patients with IBS and controls $(P=0.090$, Table 3$)$. The expression of TLR-4 and TLR-5 was 1.2-folds and 6-folds higher among patients with IBS than controls $(P=0.013$ and $P<0.001$, respectively) (Table 3). Among different subtypes of IBS, the mRNA levels of TLR-4 and TLR-5 were 1.3-folds and 8-folds higher in patients with IBS-D than controls (Table 4 and Fig.1B and 1C). Moreover, the expression of TLR-4 and TLR-5 was 6-folds and 2.7-folds higher among patients with IBS-D than IBS-C $(P=$ 0.013 and $P=0.040$, respectively). A 2-folds higher expression of TLR-5 was found in patients with IBS-C than controls $(P=$ $0.021)$, while the TLR-4 level was comparable in IBS-C patients as compared to controls $(P=0.239)$ (Fig. 1).

\section{The Protein Level of Toll-like Receptors 4 and 5 Among Patients With Irritable Bowel Syndrome and Controls}

The mRNA levels of TLR-4 and TLR-5 were further confirmed at the protein level in colonic biopsies using immunohistochemistry. Protein expressions of TLR-4 and TLR-5 were more intense in crypt and luminal surface among patients with IBS than controls (Fig. 2A and 2B). Moreover, TLR-4 expression was more prominent and 4.2-folds higher in patients with IBS-D than controls (Fig. 2C). Likewise, TLR-5 expression was 4.1-folds higher in IBS-C and 6.6-folds in IBS-D patients than controls (Fig. 2D). No positive staining was seen in the negative controls (Fig. 2A and 2B).

Table 3. Relative Expression $\left(2^{-\Delta \mathrm{Ct}}\right)$ of Toll-like Receptors, Cytokines, and Chemokines Between Patients With Irritable Bowel Syndrome and Controls

\begin{tabular}{lllr}
\hline Target gene & $\begin{array}{c}\text { Controls (n=25) } \\
\text { median (range) }\end{array}$ & $\begin{array}{c}\text { IBS (n=47) } \\
\text { median (range) }\end{array}$ & \multicolumn{1}{c}{$P$-value } \\
\hline TLR-2 & $3.00(0.0-21.0)$ & $5.00(0.0-17.0)$ & 0.090 \\
TLR-4 & $0.03(0.0-13.1)$ & $0.24(0.0-26.6)$ & 0.013 \\
TLR-5 & $0.03(0.0-1.5)$ & $0.77(0.0-6.5)$ & $<0.001$ \\
IL-6 & $0.10(0.0-2.8)$ & $0.90(0.0-5.0)$ & 0.003 \\
IL-10 & $1.00(0.0-8.1)$ & $0.60(0.0-17.2)$ & 0.546 \\
CXCL-11 & $0.10(0.0-15.4)$ & $2.80(0.0-98.8)$ & $<0.001$ \\
CXCR-3 & $0.00(0.0-25.2)$ & $1.80(0.0-24.8)$ & $<0.001$ \\
\hline
\end{tabular}

${ }^{a}$ Mann-Whitney $U$ tests. $P<0.005$ was considered significant.

IBS, irritable bowel syndrome; TLR, toll-like receptor; CXCL-11, C-X-C motif chemokine ligand 11; CXCR-3, C-X-C motif chemokine receptor 3 . 
Table 4. Relative Expression $\left(2^{-\Delta \mathrm{Ct}}\right)$ of Toll-like Receptors, Cytokines, and Chemokines, Among Different Subtypes of Patients With Irritable Bowel Syndrome and Controls

\begin{tabular}{|c|c|c|c|c|c|}
\hline Target gene & $\begin{array}{c}\text { Controls }(\mathrm{n}=25) \\
\text { median (range) }\end{array}$ & $\begin{array}{l}\text { IBS-C }(\mathrm{n}=20) \\
\text { median (range) }\end{array}$ & $\begin{array}{l}\text { IBS-D }(\mathrm{n}=20) \\
\text { median (range) }\end{array}$ & $\begin{array}{l}\text { IBS-U }(n=7) \\
\text { median (range) }\end{array}$ & $P$-value ${ }^{\mathrm{a}}$ \\
\hline TLR- $4^{b, c}$ & $0.03(0.0-13.1)$ & $0.07(0.0-5.1)$ & $0.40(0.0-26.6)$ & $0.24(0.0-2.0)$ & 0.009 \\
\hline TLR-5 ${ }^{\mathrm{b}, \mathrm{c}}$ & $0.03(0.0-1.5)$ & $0.45(0.0-4.9)$ & $2.13(0.0-6.5)$ & $2.95(0.4-4.7)$ & $<0.001$ \\
\hline IL-6 ${ }^{\mathrm{b}}$ & $0.10(0.0-2.8)$ & $0.45(0.0-5.0)$ & $1.10(0.0-2.1)$ & $0.90(0.0-1.7)$ & 0.016 \\
\hline IL-10 ${ }^{\mathrm{b}, \mathrm{c}}$ & $1.00(0.0-8.1)$ & $2.25(0.0-17.2)$ & $0.05(0.0-8.8)$ & $0.00(0.0-3.0)$ & 0.003 \\
\hline CXCL-1 $11^{\mathrm{b}}$ & $0.10(0.0-15.4)$ & $1.35(0.0-34.7)$ & $3.60(0.0-57.9)$ & $29.00(0.4-98.8)$ & $<0.001$ \\
\hline CXCR-3 $3^{\mathrm{b}, \mathrm{c}, \mathrm{d}}$ & $0.00(0.0-25.2)$ & $0.80(0.0-8.3)$ & $4.40(0.0-24.8)$ & $1.00(0.3-12.0)$ & $<0.001$ \\
\hline
\end{tabular}

${ }^{\mathrm{a}}$ Kruskal Wallis test with post hoc analysis. $P<0.05$ was considered significant.

${ }^{\mathrm{b}} P<0.017$ between patients with IBS-D and controls.

${ }^{c} P<0.017$ between IBS-C and IBS-D.

${ }^{\mathrm{d}}$ Bonferroni corrected $P$-value $<0.017$ was considered significant for subgroup analysis.
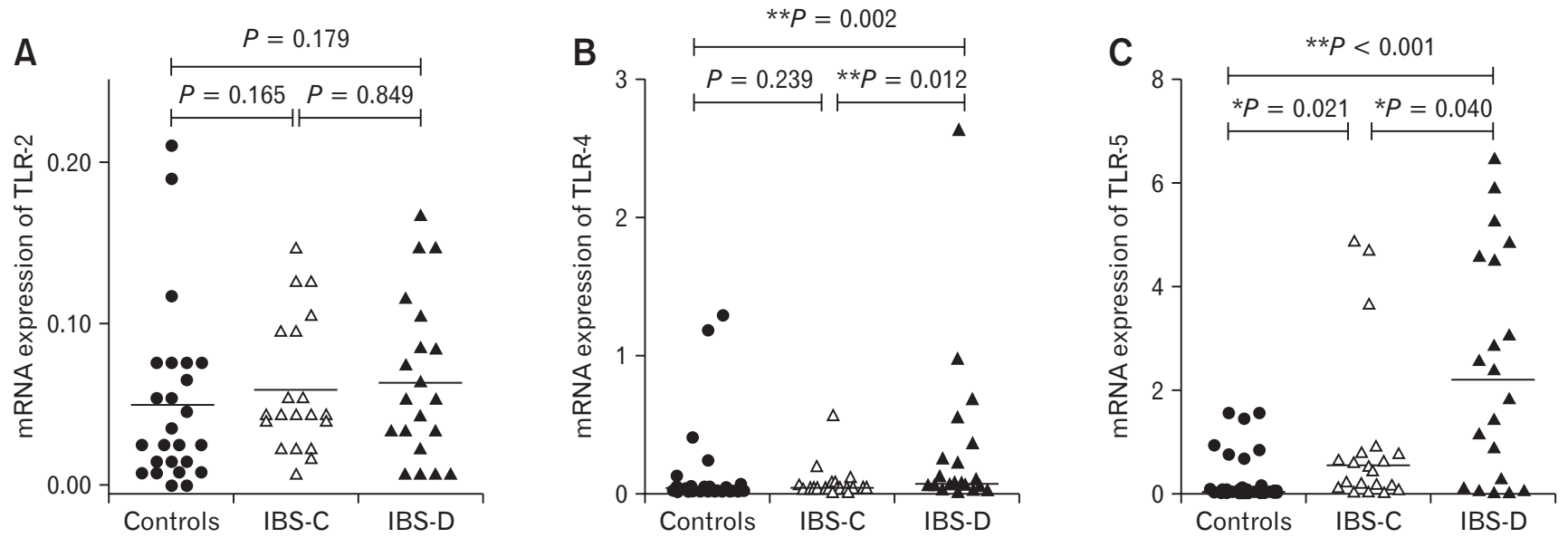

Figure 1. Relative expression of toll-like receptors (TLRs) among different subtypes of patients with irritable bowel syndrome (IBS) and controls. (A) TLR-2, (B) TLR-4, and (C) TLR-5. Mann-Whitney $U$ test was used for pair-wise comparison of mRNA level of TLRs (2, 4, and 5) among different subtypes of IBS and controls. Post hoc Bonferroni test was applied for multiple comparisons. ${ }^{*} P$-values $<0.05$ were considered as significant. ${ }^{*}$ Bonferroni corrected $P$-values $<0.017$ were considered as significant for subgroup analysis. IBS-C, constipation-predominant IBS; IBSD, diarrhea-predominant IBS.

\section{The Messenger RNA Levels of Cytokines} (Interleukin-6 and Interleukin-10), Chemokine C-X-C Motif Chemokine Ligand 11 and Its Receptor (C-X-C Motif Chemokine Receptor 3)

The mRNA level of IL-6 was 2.2-folds higher among patients with IBS than controls $(P=0.003)$. Likewise, CXCL-11 and its receptor CXCR-3 were 10-folds and 1.5-folds higher among patients with IBS than controls $(P<0.001$ and $P<0.001$, respectively). Among the different subtypes of IBS, mRNA level of IL-6 was 2 -folds higher in patients with IBS-D than controls $(P=0.002)$
(Table 4). Likewise, CXCL-11 and CXCR-3 were up-regulated in IBS-D patients as compared to controls (Fig. 3C and 3D). Moreover, mRNA level of CXCR-3 was 4-folds higher among patients with IBS-D than IBS-C (Table 4).

There was no difference in mRNA level of an anti-inflammatory cytokine (IL-10) in patients with IBS than controls $(P=0.546)$. Expression of IL-10 was down-regulated among patients with IBS-D than IBS-C and controls (Fig. 3B). The mRNA levels of TLRs, cytokines, and chemokines among different subtypes of IBS and controls are presented in Table 4. 


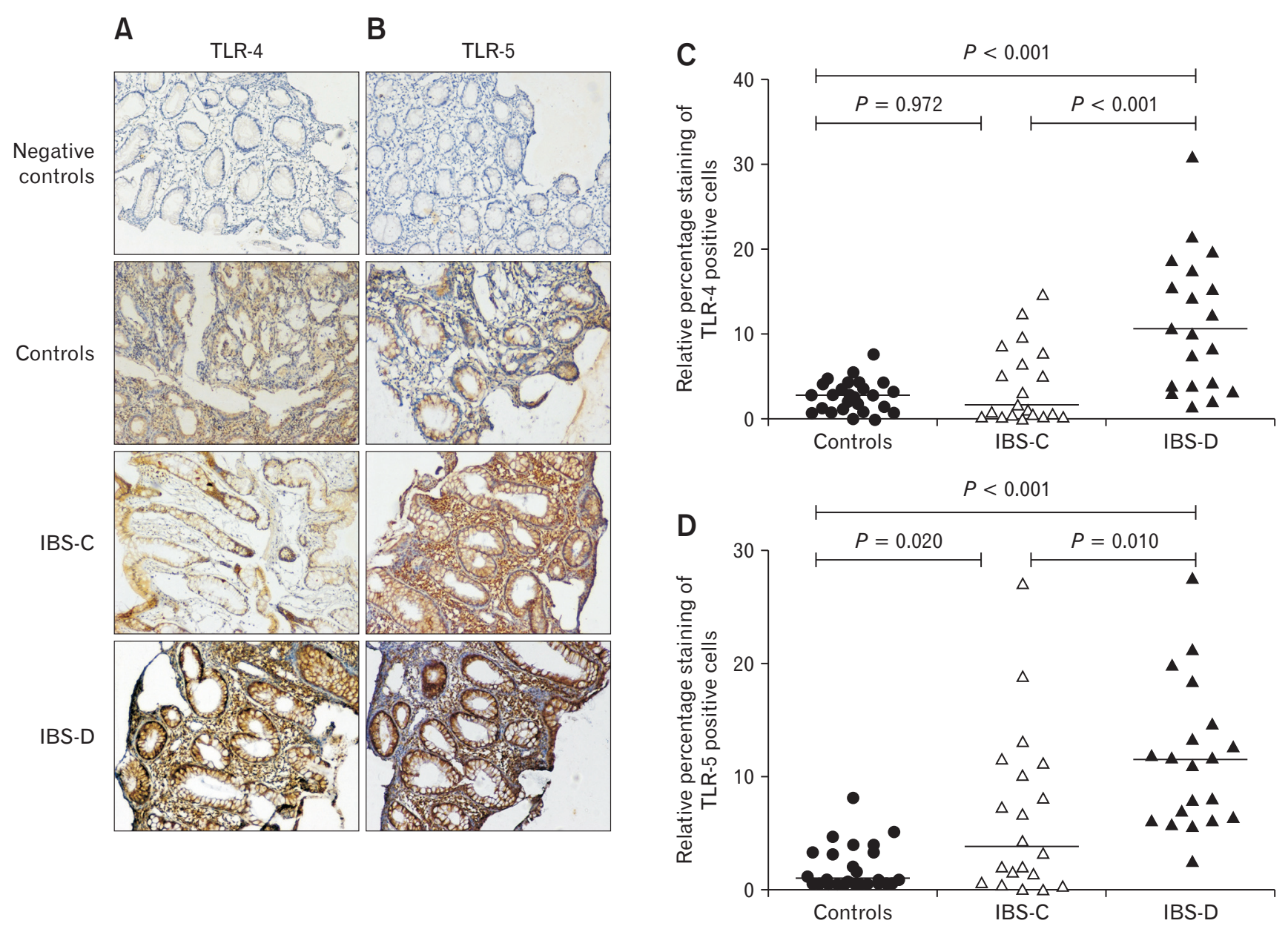

Figure 2. Immunohistochemical analysis of Toll-like receptors (TLRs) 4 and 5 among different subtypes of patients with irritable bowel syndrome (IBS) and controls. Figures (A) and (B) show protein expression of TLR-4 and TLR-5 in colonic biopsies among different subtypes of IBS patients and controls. Protein expression of TLR-4 (C) and TLR-5 (D) was quantified using Image J software plugins. Figures (C) and (D) show the percentage contribution of positive cells for TLR-4 and TLR-5. IBS-C, constipation-predominant IBS; IBS-D, diarrhea-predominant IBS.

\section{Correlation Between Messenger RNA Level of Toll- like Receptors, Cytokines, and Chemokines}

There was a trend towards a correlation between mRNA levels of TLR-4 and TLR-5 (Spearman correlation coefficient $[r]=$ $0.284, P=0.053$ ) among patients with IBS (Fig. 4A). A positive correlation was seen between mRNA level of TLR-4 with IL-6 $(r$ $=0.296, P=0.043$ ) (Fig. 4B). Moreover, mRNA expression of CXCR-3 showed correlation with CXCL-11 and IL-6 ( $r=0.290$, $P=0.047$ and $r=0.423, P=0.003$, respectively) (Fig. $4 \mathrm{C}$ and 4D).

\section{Association Between Symptoms of Irritable Bowel Syndrome and Messenger RNA Levels of Toll-like Receptors, Cytokines, and Chemokines}

The expressions of TLR-4, TLR-5 and CXCR-3 were higher among IBS patients with loose watery stool with frequency more than 3 times per day than those without (Table 5). Moreover, the mRNA level of IL-10 was lower in patients who had more frequent and loose stool at the onset of pain, stool frequency more than 3 times per day than those without (Table 5). In contrast, patients presenting with hard or lumpy stool had a reduced level of TLR-4 and CXCR-3 and increased level of IL-10 than those without (Table $5)$. 

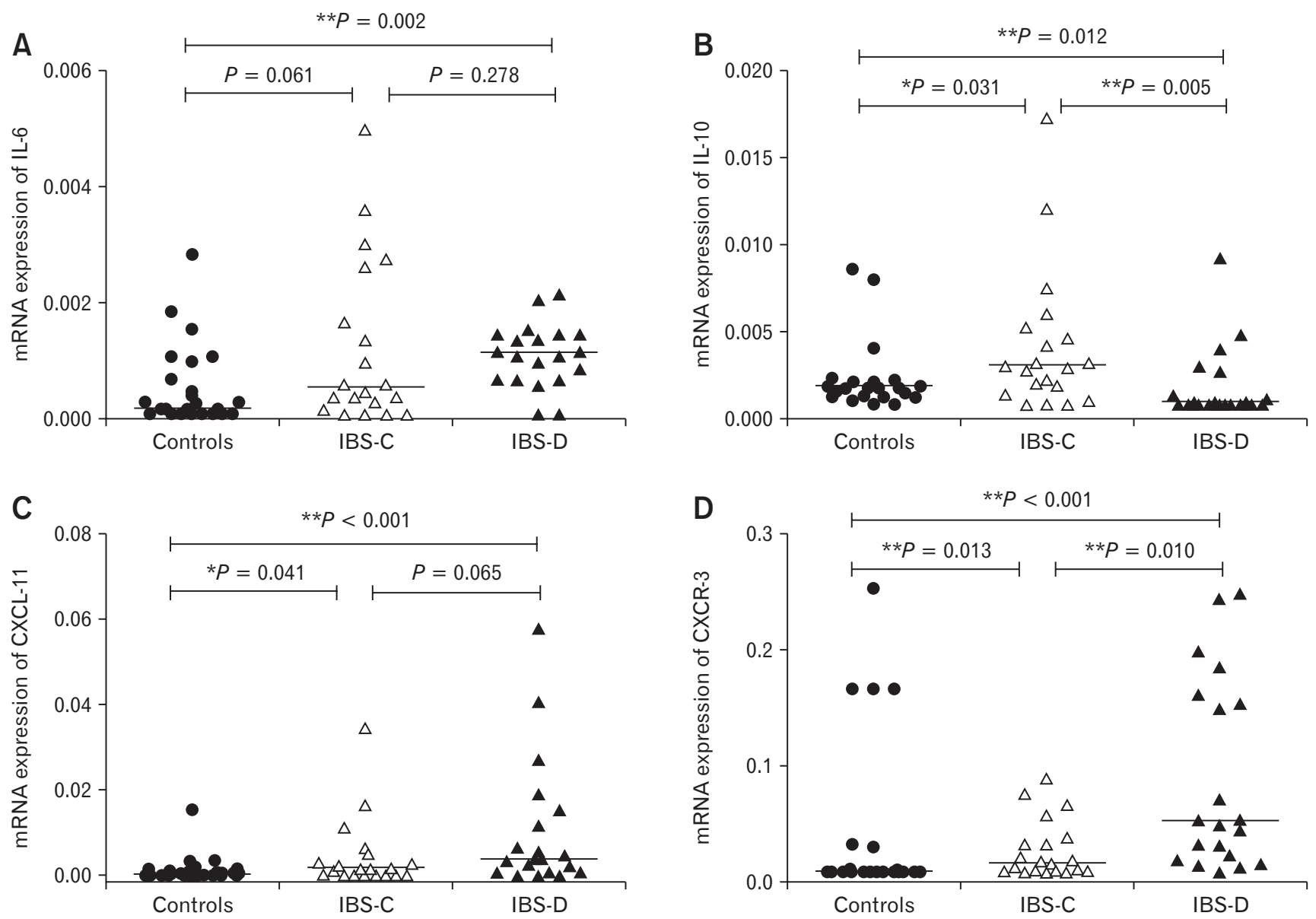

Figure 3. Comparison of cytokines and chemokines among different subtypes of patients with irritable bowel syndrome (IBS) and controls. (A) IL-6, (B) IL-10, (C) C-X-C motif chemokine ligand 11 (CXCL-11), and (D) C-X-C motif chemokine receptor 3 (CXCR-3). Mann-Whitney $U$ test was used for pair-wise comparison of mRNA levels of cytokines (IL-6 and IL-10) and chemokines (CXCL-11 and CXCR-3) among different subtypes of irritable bowel syndrome (IBS) and controls. Post hoc Bonferroni test was applied for multiple comparisons. ${ }^{*} P$-values $<0.05$ were considered as significant. ${ }^{*}$ Bonferroni corrected $P$-values $<0.017$ were considered as significant for subgroup analysis. IBS-C, constipationpredominant IBS; IBS-D, diarrhea-predominant IBS.

\section{Correlation Between Messenger RNA Levels of Toll- like Receptors, Cytokines, and Chemokines With Duration of Symptoms and Weekly Stool Frequency}

The mRNA expression of TLR-4 and CXCR-3 showed correlation with weekly stool frequency in IBS patients $(r=0.349$, $P=0.016$ and $r=0.399, P=0.005$, respectively) (Fig. $5 \mathrm{~A}$ and $5 \mathrm{C})$. Moreover, expression of IL-10 inversely correlated with stool frequency $(r,-0.435 ; P=0.002)$ (Fig. 5B). However, there was no correlation between duration of symptoms and mRNA expression of TLRs, cytokines, and chemokines (Table 6).

\section{Correlation Between the Copy Number of Altered Fecal Microbiota and Messenger RNA Levels of Toll- like Receptors, Cytokines, and Chemokines}

The number of copies of Lactobacillus and Bifidobacterium species correlated with IL-10 level among patients with IBS-C ( $r=0.453, P=0.045$ and $r=0.554, P=0.011$, respectively). Moreover, number of copies of Gram-positive and Gram-negative bacteria showed correlation with CXCL-11 $(r=0.482, P=0.031$ and $r=0.561, P=0.010$, respectively) among patients with IBSD. 

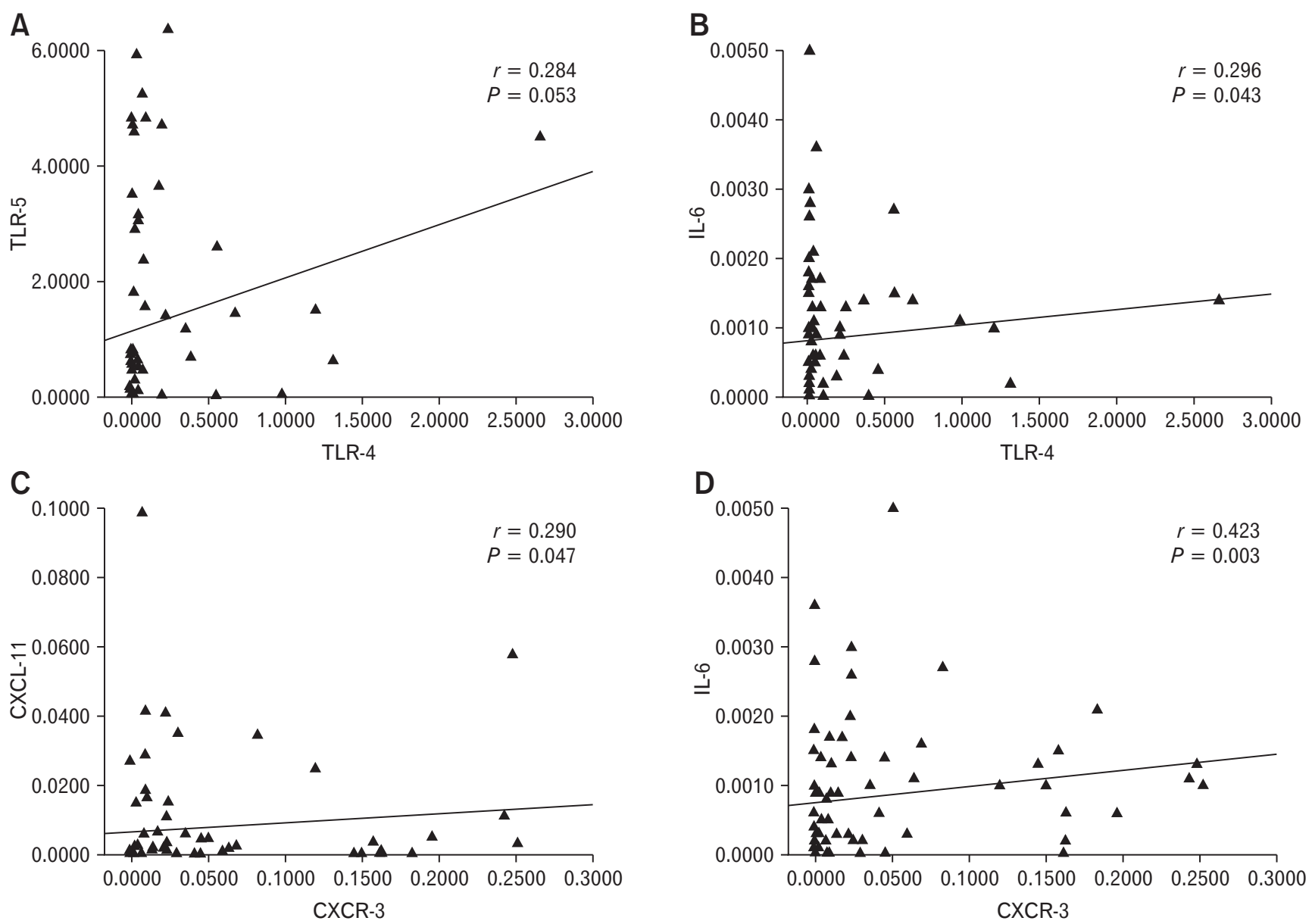

Figure 4. Correlation among toll-like receptors (TLRs), cytokines, and chemokines using nonparametric Spearman correlation. (A) Between TLR-4 and TLR-5, (B) TLR-4 and IL-6, (C) C-X-C motif chemokine receptor 3 (CXCR-3) and C-X-C motif chemokine ligand 11 (CXCL-11), and (D) CXCR-3 and IL-6.

\section{Discussion}

Recent studies show that low-grade inflammation in response to altered gut microbiota may be involved in the pathophysiology of IBS. The present study suggests that TLR-mediated cytokines and chemokines may play important role in the pathophysiology of mild inflammation at least in a subgroup of IBS patients with following evidence: (1) up-regulation of TLR-4 and TLR-5 among patients with IBS, particularly IBS-D, than controls, (2) expression of proinflammatory cytokine (IL-6) was higher, while anti-inflammatory cytokine (IL-10) lower in IBS-D patients than controls, (3) chemokines CXCL-11 and its receptor CXCR-3 were higher in IBS$\mathrm{D}$ patients than controls, (4) mRNA level of TLR-4 showed correlation with IL-6; CXCR-3 showed correlation with CXCL-11 and IL-6 among patients with IBS, and (5) mRNA expression of
TLR-4 and CXCR-3 showed correlation with stool frequency per week, while IL-10 inversely correlated with stool frequency in IBS patients.

IECs act as a barrier between luminal microbiota and gutassociated immune cells. ${ }^{30}$ In vitro, in vivo, and several intestinal epithelial cell lines express TLRs. ${ }^{31}$ TLRs play a pivotal role in maintaining the gut homeostasis through secretion of pro-inflammatory cytokines and chemokines. ${ }^{32}$ Altered expressions of TLRs in response to dysbiosis have been reported in several GI diseases. ${ }^{12}$ Moreover, one study from our center showed that Gram-negative bacteria (particularly Pseudomonas aeruginosa and Bacteroides thetaiotamicron) and segmented filamentous bacteria were increased, while Gram-positive bacteria were comparable among patients with IBS-D than controls. ${ }^{3}$ TLR-4 and TLR-5 show activity against the ligands of Gram-negative (lipopolysaccharides) and segmented filamentous bacteria (flagellin protein). ${ }^{4}$ Therefore, we studied the role 


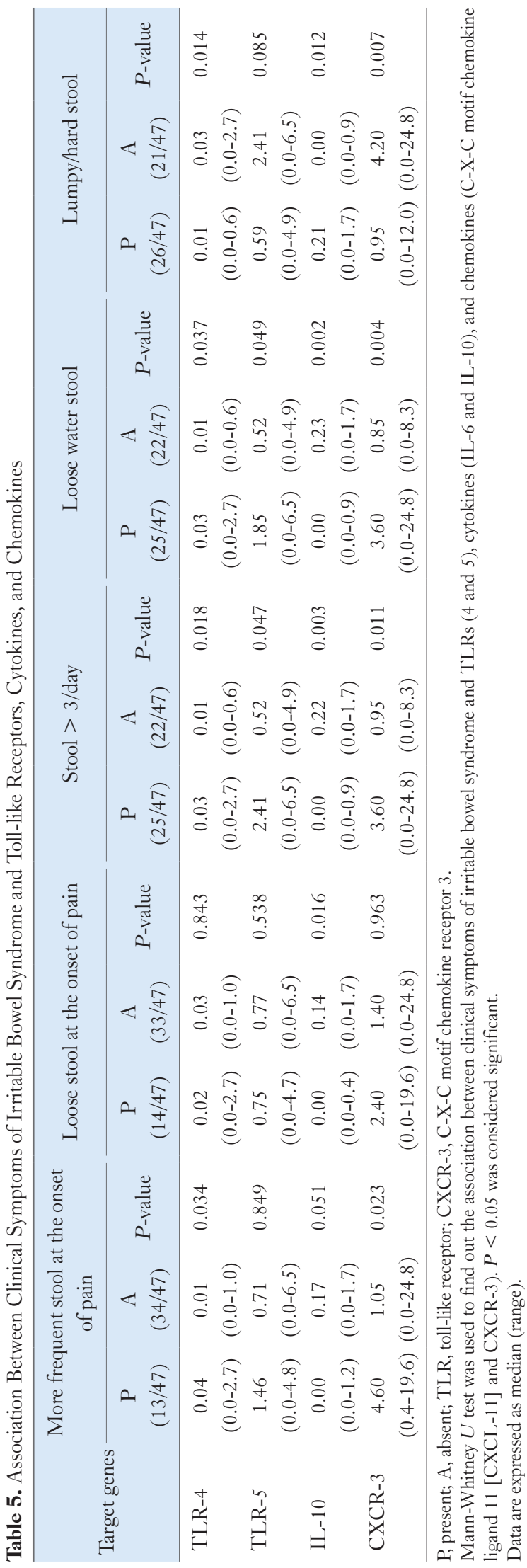



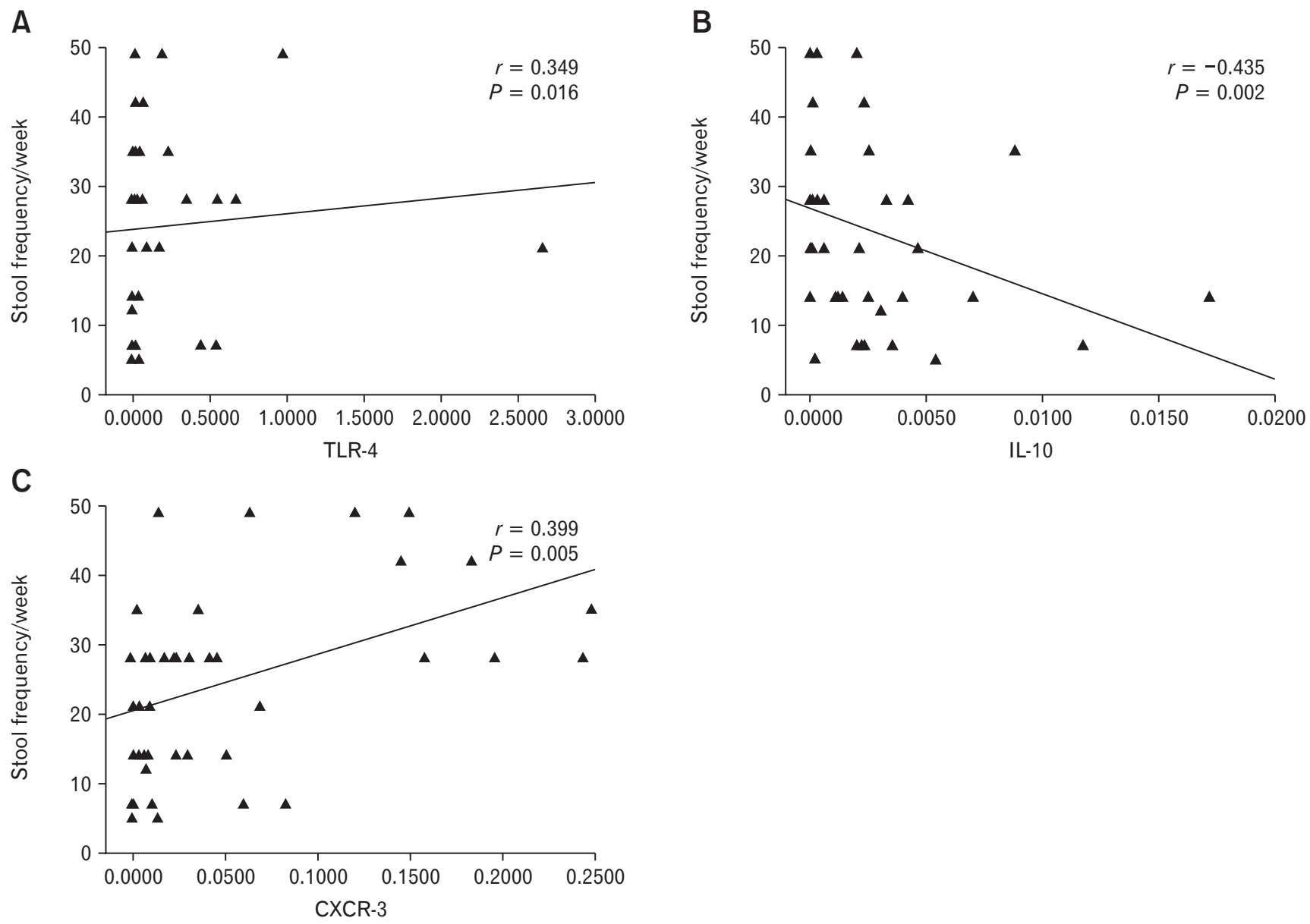

Figure 5. Spearman correlation of toll-like receptor-4 (TLR-4), IL-10, and C-X-C motif chemokine receptor 3 (CXCR-3) with stool frequency per week. Stool frequency per week showed a correlation with TLR-4 and CXCR-3 (A, B), while negative correlation with IL-10 (C).

of TLR-4 and TLR-5 in the pathogenesis of low-grade inflammation among patients with IBS.

In the present study, mRNA level of TLR-2 was comparable among patients with IBS and controls, which is in accordance with a previous study. ${ }^{4}$ We found mRNA and protein levels of TLR-4 and TLR-5 were up-regulated among patients with IBS, particularly IBS-D, than controls supporting the hypothesis that mild inflammation occurs in a subtype of IBS. Our results corroborated with a higher level of TLR-4 and TLR-5 reported in patients with IBS. ${ }^{4}$ Moreover, up-regulation of TLR-4 was seen in patients with Crohn's disease and ulcerative colitis. ${ }^{31} \mathrm{~A}$ study showed that rats with stress-induced IBS had higher mRNA levels of TLR-4 and TLR-5 in colonic mucosa than controls. ${ }^{33}$ Likewise, another study reported that the expression of TLR-4 and TLR-5 was 4-folds and 1.7-folds higher among female IBS patients as compared to controls. ${ }^{4}$ Results of immunohistochemistry showed that expression of TLR-4 and TLR-5 was more prominent in the colonic crypt as well as in intestinal epithelial cells, which is in accordance with a previous study. ${ }^{4}$ Conversely, Belmonte and his colleagues ${ }^{8}$ showed that mRNA and protein levels of TLR-4 in colonic mucosa were comparable in IBS-D, while higher in IBS-M patients as compared to controls. This may be due to heterogeneity in IBS patients and control population as well as the site of sample collection. ${ }^{8}$

TLRs play an important role in the activation of cytokine and chemokine release and its higher levels have been reported in patients with IBD and IBS. ${ }^{4,12,34-36}$ Recently, a study showed a higher level of IL-6 in the peripheral blood of IBS patients on stimulation with TLR-ligands compared to controls, which is in accordance with the present study. ${ }^{12}$ Though several studies reported IL-6 expression in peripheral blood of patients with IBS, its level in colonic biopsies is scanty. IL-6 is an important pro-inflammatory cytokine and its alteration may lead to variation in the level of GI mucosal inflammation and hence, the severity of IBS symptoms. ${ }^{37-39}$ In addition, the mRNA level of TLR-4 showed a positive correlation with 
Table 6. Correlation Analysis of Toll-like Receptors, Cytokines, and Chemokines With Duration of Symptoms and Stool Frequency per Week

\begin{tabular}{ccc}
\hline Target gene & $\begin{array}{c}\text { Duration of } \\
\text { symptoms }\end{array}$ & $\begin{array}{c}\text { Stool } \\
\text { frequency/wk }\end{array}$ \\
\hline TLR-2 & & \\
$r$ & -0.262 & -0.132 \\
$P$-value & 0.076 & 0.376 \\
TLR-4 & & \\
$r$ & -0.144 & 0.349 \\
$P$-value & 0.333 & 0.016 \\
TLR-5 & & \\
$r$ & -0.154 & 0.254 \\
$P$-value & 0.302 & 0.085 \\
IL-6 & & 0.133 \\
$r$ & 0.038 & 0.374 \\
$P$-value & 0.799 & -0.435 \\
IL-10 & & 0.002 \\
$r$ & 0.058 & 0.129 \\
$P$-value & 0.699 & 0.387 \\
CXCL-11 & & 0.005 \\
$r$ & 0.246 & \\
$P$-value & 0.096 & \\
CXCR-3 & 0.031 & \\
$r$ & 0.835 & \\
$P$-value & & \\
\hline to- & & \\
\hline
\end{tabular}

TLR, toll-like receptor; CXCL-11, C-X-C motif chemokine ligand 11; CXCR-3, C-X-C motif chemokine receptor 3.

$r$, Spearman correlation coefficient. $P<0.05$ was considered significant.

TLR-5 and IL-6 in the present study. These findings indicate that TLRs act synergistically to stimulate the innate immune response and subsequent release of pro-inflammatory cytokines and chemokines in response to gut dysbiosis.

We found that the mRNA level of an anti-inflammatory cytokine (IL-10) was reduced in patients with IBS-D than controls. This finding corroborated with the results of previous studies showing a reduced level of IL-10 in the sigmoid colon in female IBS patients than controls. ${ }^{40}$ Recently, another study showed reduced expression of IL-10 in colonic biopsies among patients with IBSD than controls. ${ }^{41}$ The key role of IL-10 is to inhibit the synthesis of pro-inflammatory cytokines. But, its reduced level in the intestine favors synthesis and secretion of inflammatory cytokines and chemokines that are in agreement with the present findings. At the genotypic level, van der Veek et $\mathrm{al}^{42}$ showed that the low producer IL-10 genotype was more frequent among patients with IBS than controls. Thus, reduced level of IL-10 suggests that patients with IBS are more susceptible to external stimuli (gut pathogens and antigens) causing inflammation as compared to controls.

The role of chemokines against luminal antigens in the pathogenesis of IBS is unknown. Published data showed that exposure of TLR-5 ligands led to higher levels of CXCL-11 in serum as well as in biopsies among patients with IBD. ${ }^{16}$ Moreover, the CXCR-3/ CXCL-11 pathway is involved in response to bacterial flagellin in several intestinal immune mediated disorders like IBD. ${ }^{16} \mathrm{CXCR}$ 3 is mainly expressed by Th1 cells. ${ }^{43}$ Therefore, binding of CXCL11 to CXCR-3 may stimulate Th-1 cells to release IL-6. ${ }^{16}$ In the present study, we also found a positive correlation between mRNA levels of CXCR-3 and IL-6. In support of this, a study showed that CXCR-3 inhibition reduced the expression of IL-6 in patients with IBD. ${ }^{17,44}$ Alterations in the level of cytokines and chemokines among IBS patients may be due to increased permeability of IECs resulting in translocation of colonic bacteria to the underlying submucosa. ${ }^{4}$ In addition, leaky gut epithelium and down- regulation of tight junction proteins such as occludin have been reported in IBS patients. ${ }^{45}$ These data suggest that CXCR-3/CXCL-11 play a substantial role in the pathogenesis of low-grade inflammation, at least in a subgroup of patients with IBS.

We found IBS patients with more frequent and loose stool at the onset of pain had increased level of TLR-4 and decreased level of IL-10 than those without. Moreover, a positive correlation was found between mRNA levels of TLR-4 and CXCR-3 with weekly stool frequency in IBS patients. In contrast, a previous study, ${ }^{8}$ failed to show a correlation between mRNA levels of TLRs and duration of symptoms. Recent studies reported that higher level of cytokines resulted in visceral hyperalgesia ${ }^{12,46}$ and stress response, which might affect GI motility in IBS patients. ${ }^{47}$ Moreover, patients with IBS express hypersensitivity to pain. ${ }^{12}$

We wish to emphasize that alteration in gut microbiota may have a crucial role in the pathogenesis of low-grade inflammation in patients with IBS. A positive correlation was found between the copy number of Lactobacillus and Bifidobacterium species and mRNA levels of IL-10 among patients with IBS-C. Recent studies have shown that the number of copies of Lactobacillus and Bifidobacterium was reduced in IBS patients than controls. ${ }^{3,48,49}$ A study has shown that administration of Lactobacillus rhamnosus GR-1 and Lactobacillus reuteri $\mathrm{RC}-14$ enhances the population of Treg cells in patients with IBS. ${ }^{50}$ Moreover, administration of Bifidobacterium infantis 35624 increased the level of anti-inflammatory cytokines and normalized the ratio of IL-10/IL-12. ${ }^{51}$ Thus, the reduction in a number of Lactobacillus and Bifidobacterium may be associated with low-grade inflammation. In the present study, Gram-positive and Gram-negative bacteria showed a correlation 
with CXCL-11 among patients with IBS-D. In support of this, higher copy number of Gram-negative bacteria particularly Bacteroides species and $P$. aeruginosa has been reported among patients with IBS-D than controls. ${ }^{3}$ To the best of our knowledge, this is the first study evaluating the correlation between altered fecal microbiota and mRNA levels of immune mediators among different subtypes of IBS patients. Manipulation of gut microbiota using probiotic, antibiotics, and fecal transplantation may be useful in IBS and IBD patients, further proving the role of dysbiosis and inflammation in the pathogenesis of a subset of patients. ${ }^{52-54}$

In conclusion, up-regulation of TLR-4 and TLR-5 suggest that the innate immune system may play an important role in the pathophysiology of IBS. The up-regulation of pro-inflammatory cytokines (IL-6, CXCL-11, and CXCR-3) and down-regulation of the anti-inflammatory cytokine (IL-10) in patients with IBS-D than controls suggest the presence of low-grade intestinal inflammation in a subgroup of IBS patients. This study shows a relationship between the mRNA of TLRs, cytokines, chemokines, and copy number of altered fecal microbiota. These results highlight the complex interactions between the host immune system and gut microbiota leading to the pathogenesis of intestinal inflammation. Thus, alteration in normal microbial flora seems to contribute to dysregulated immune response among patients with IBS.

Acknowledgements: We would like to thank all the recruited subjects and volunteers for taking part in this study. This study was presented in Digestive Disease Week, Chicago, IL, May 8, 2017 (Abstract \#2681616) and 58th Annual Conference of the Indian Society of Gastroenterology, Bubaneswar on 14th-17th December, 2017.

Financial support: Ratnakar Shukla acknowledges the financial assistance received from Department of Science and Technology, Government of India (Grant No. DST/INSPIRE Fellowship/2010/IF1 10001).

\section{Conflicts of interest: None.}

Author contributions: Ratnakar Shukla: execution of laboratory works, data analysis, and drafting of the manuscript; Prabhat Ranjan: laboratory works and critical revision of the manuscript; Ujjala Ghoshal: concept of the study, supervision of laboratory works, and critical revision of the manuscript; and Uday C Ghoshal: study concept, clinical works, data analysis, and critical revision of the manuscript.

\section{References}

1. Ghoshal UC, Shukla R, Ghoshal U, Gwee KA, Ng SC, Quigley EM. The gut microbiota and irritable bowel syndrome: friend or foe? Int J Inflam 2012;2012:151085.

2. Ghoshal UC, Abraham P, Bhatt C, et al. Epidemiological and clinical profile of irritable bowel syndrome in India: report of the Indian society of gastroenterology task force. Indian J Gastroenterol 2008;27:22-28.

3. Shukla R, Ghoshal U, Dhole TN, Ghoshal UC. Fecal microbiota in patients with irritable bowel syndrome compared with healthy controls using real-time polymerase chain reaction: an evidence of dysbiosis. Dig Dis Sci 2015;60:2953-2962.

4. Brint EK, MacSharry J, Fanning A, Shanahan F, Quigley EM. Differential expression of toll-like receptors in patients with irritable bowel syndrome. Am J Gastroenterol 2011;106:329-336.

5. Hajela N, Ramakrishna BS, Nair GB, Abraham P, Gopalan S, Ganguly NK. Gut microbiome, gut function, and probiotics: implications for health. Indian J Gastroenterol 2015;34:93-107.

6. Öhman L, Törnblom H, Simrén M. Crosstalk at the mucosal border: importance of the gut microenvironment in IBS. Nat Rev Gastroenterol Hepatol 2015;12:36-49.

7. Ortiz-Lucas M, Saz-Peiró P, Sebastián-Domingo JJ. Irritable bowel syndrome immune hypothesis. Part two: the role of cytokines. Rev Esp Enferm Dig 2010;102:711-717.

8. Belmonte L, Beutheu Youmba S, Bertiaux-Vandaële N, et al. Role of toll like receptors in irritable bowel syndrome: differential mucosal immune activation according to the disease subtype. PLoS One 2012;7:e42777.

9. Abreu MT. Toll-like receptor signalling in the intestinal epithelium: how bacterial recognition shapes intestinal function. Nat Rev Immunol 2010;10:131-144.

10. Gribar SC, Anand RJ, Sodhi CP, Hackam DJ. The role of epithelial tolllike receptor signaling in the pathogenesis of intestinal inflammation. J Leukoc Biol 2008;83:493-498.

11. Cario E. Bacterial interactions with cells of the intestinal mucosa: toll-like receptors and NOD2. Gut 2005;54:1182-1193.

12. McKernan DP, Gaszner G, Quigley EM, Cryan JF, Dinan TG. Altered peripheral toll-like receptor responses in the irritable bowel syndrome. Aliment Pharmacol Ther 2011;33:1045-1052.

13. Frosali S, Pagliari D, Gambassi G, Landolfi R, Pandolfi F, Cianci R. [How the intricate interaction among toll-like receptors, microbiota, and intestinal immunity can influence gastrointestinal pathology.] J Immunol Res 2015;2015:489821. [Chinese]

14. Dillon S, Agrawal A, Van Dyke T, et al. A toll-like receptor 2 ligand stimulates Th2 responses in vivo, via induction of extracellular signalregulated kinase mitogen-activated protein kinase and c-fos in dendritic cells. J Immunol 2004;172:4733-4743.

15. Wong CK, Wong PT, Tam LS, Li EK, Chen DP, Lam CW. Activation profile of toll-like receptors of peripheral blood lymphocytes in patients with systemic lupus erythematosus. Clin Exp Immunol 2010;159:11-22.

16. Liu Z, Chen X, Wang X, et al. Chemokine CXCL11 links microbial stimuli to intestinal inflammation. Clin Exp Immunol 2011;164:396-406. 
17. Tokuyama H, Ueha S, Kurachi M, et al. The simultaneous blockade of chemokine receptors CCR2, CCR5 and CXCR3 by a non-peptide chemokine receptor antagonist protects mice from dextran sodium sulfatemediated colitis. Int Immunol 2005;17:1023-1034.

18. Danese S, Gao B. Interleukin-6: a therapeutic Jekyll and Hyde in gastrointestinal and hepatic diseases. Gut 2010;59:149-151.

19. Bashashati M, Rezaei N, Shafieyoun A, et al. Cytokine imbalance in irritable bowel syndrome: a systematic review and meta-analysis. Neurogastroenterol Motil 2014;26:1036-1048.

20. Ghoshal UC, Gwee KA, Chen M, et al. Development, translation and validation of enhanced asian rome III questionnaires for diagnosis of functional bowel diseases in major asian languages: a rome foundationasian neurogastroenterology and motility association working team report. J Neurogastroenterol Motil 2015;21:83-92.

21. Xu J, Yang Y, Sun J, et al. Expression of toll-like receptors and their association with cytokine responses in peripheral blood mononuclear cells of children with acute rotavirus diarrhoea. Clin Exp Immunol 2006;144:376-381.

22. Schaefer TM, Desouza K, Fahey JV, Beagley KW, Wira CR. toll-like receptor (TLR) expression and TLR-mediated cytokine/chemokine production by human uterine epithelial cells. Immunology 2004;112:428436.

23. Jin SH, Kang HY. Activation of toll-like receptors 1, 2, 4, 5, and 7 on human melanocytes modulate pigmentation. Ann Dermatol 2010;22:486489 .

24. Zhou Y, Wei Y, Wang L, et al. Decreased adiponectin and increased inflammation expression in epicardial adipose tissue in coronary artery disease. Cardiovasc Diabetol 2011;10:2.

25. Hida N, Shimoyama T Jr, Neville P, et al. Increased expression of IL10 and IL-12 (p40) mRNA in Helicobacter pylori infected gastric mucosa: relation to bacterial cag status and peptic ulceration. J Clin Pathol 1999;52:658-664.

26. Schroepf S, Kappler R, Brand S, et al. Strong overexpression of CXCR3 axis components in childhood inflammatory bowel disease. Inflamm Bowel Dis 2010;16:1882-1890.

27. Kimang'a A, Revathi G, Kariuki S, et al. IL-17A and IL-17F gene expression is strongly induced in the mucosa of $H$. pylori-infected subjects from Kenya and Germany. Scand J Immunol 2010;72:522-528.

28. Stanislawowski M, Wierzbicki PM, Golab A, et al. Decreased toll-like receptor-5 (TLR-5) expression in the mucosa of ulcerative colitis patients. J Physiol Pharmacol 2009;60(suppl 4):71-75.

29. Liang WJ, Zhang G, Luo HS, Liang LX, Huang D, Zhang FC. Tryptase and protease-activated receptor 2 expression levels in irritable bowel syndrome. Gut Liver 2016;10:382-390.

30. Ipci K, Altintoprak N, Muluk NB, Senturk M, Cingi C. The possible mechanisms of the human microbiome in allergic diseases. Eur Arch Otorhinolaryngol 2017;274:617-626.

31. Cario E, Podolsky DK. Differential alteration in intestinal epithelial cell expression of toll-like receptor 3 (TLR3) and TLR4 in inflammatory bowel disease. Infect Immun 2000;68:7010-7017.

32. Wells JM, Rossi O, Meijerink M, van Baarlen P. Epithelial crosstalk at the microbiota-mucosal interface. Proc Natl Acad Sci USA 2011;108(suppl 1):4607-4614.

33. McKernan DP, Nolan A, Brint EK, et al. Toll-like receptor mRNA expression is selectively increased in the colonic mucosa of two animal models relevant to irritable bowel syndrome. PLoS One 2009;4:e8226.

34. Means TK, Hayashi F, Smith KD, Aderem A, Luster AD. The toll-like receptor 5 stimulus bacterial flagellin induces maturation and chemokine production in human dendritic cells. J Immunol 2003;170:5165-5175.

35. Thompson-Chagoyán OC, Maldonado J, Gil A. Aetiology of inflammatory bowel disease (IBD): role of intestinal microbiota and gut-associated lymphoid tissue immune response. Clin Nutr 2005;24:339-352.

36. Darkoh C, Comer L, Zewdie G, Harold S, Snyder N, Dupont HL. Chemotactic chemokines are important in the pathogenesis of irritable bowel syndrome. PLoS One 2014;9:e93144.

37. Hughes PA, Zola H, Penttila IA, Blackshaw LA, Andrews JM, Krumbiegel D. Immune activation in irritable bowel syndrome: can neuroimmune interactions explain symptoms? Am J Gastroenterol 2013;108:1066-1074.

38. Chang L, Sundaresh S, Elliott J, et al. Dysregulation of the hypothalamic-pituitary-adrenal (HPA) axis in irritable bowel syndrome. Neurogastroenterol Motil 2009;21:149-159.

39. Jardí F, Fernández-Blanco JA, Martínez V, Vergara P. Plasticity of dorsal root ganglion neurons in a rat model of post-infectious gut dysfunction: potential implication of nerve growth factor. Scand J Gastroenterol 2014;49:1296-1303

40. Chang L, Adeyemo M, Karagiannides I, et al. Serum and colonic mucosal immune markers in irritable bowel syndrome. Am J Gastroenterol 2012;107:262-272.

41. De Silva AP, Nandasiri SD, Hewavisenthi J, et al. Subclinical mucosal inflammation in diarrhea-predominant irritable bowel syndrome (IBS) in a tropical setting. Scand J Gastroenterol 2012;47:619-624.

42. van der Veek PP, van den Berg M, de Kroon YE, Verspaget HW, Masclee AA. Role of tumor necrosis factor- $\alpha$ and interleukin-10 gene polymorphisms in irritable bowel syndrome. Am J Gastroenterol 2005;100:2510-2516.

43. Geiger B, Wenzel J, Hantschke M, Haase I, Ständer S, von Stebut E. Resolving lesions in human cutaneous leishmaniasis predominantly harbour chemokine receptor CXCR3-positive T helper 1/T cytotoxic type 1 cells. Br J Dermatol 2010;162:870-874.

44. Bao CR, Tang GY, Zhang XP, Quan ZW. Lentivirus-mediated gene transfer of small interfering RNA against the chemokine receptor CXCR3 suppresses cytokine indicators of acute graft rejection in a rat model. J Int Med Res 2010;38:1113-1120.

45. Keszthelyi D, Troost FJ, Jonkers DM, et al. Serotonergic reinforcement of intestinal barrier function is impaired in irritable bowel syndrome. Aliment Pharmacol Ther 2014;40:392-402.

46. Arebi N, Gurmany S, Bullas D, Hobson A, Stagg A, Kamm M. Review article: the psychoneuroimmunology of irritable bowel syndrome--an exploration of interactions between psychological, neurological and immunological observations. Aliment Pharmacol Ther 2008;28:830-840.

47. Taché Y, Martinez V, Wang L, Million M. CRF1 receptor signaling pathways are involved in stress-related alterations of colonic function and viscerosensitivity: implications for irritable bowel syndrome. Br J Phar- 
macol 2004;141:1321-1330

48. Malinen E, Rinttilä T, Kajander K, et al. Analysis of the fecal microbiota of irritable bowel syndrome patients and healthy controls with real-time PCR. Am J Gastroenterol 2005;100:373-382.

49. Kerckhoffs AP, Samsom M, van der Rest ME, et al. Lower Bifidobacteria counts in both duodenal mucosa-associated and fecal microbiota in irritable bowel syndrome patients. World J Gastroenterol 2009;15:28872892.

50. Borchers AT, Selmi C, Meyers FJ, Keen CL, Gershwin ME. Probiotics and immunity. J Gastroenterol 2009;44:26-46.

51. O'Mahony L, McCarthy J, Kelly P, et al. Lactobacillus and bifidobacte- rium in irritable bowel syndrome: symptom responses and relationship to cytokine profiles. Gastroenterology 2005;128:541-551.

52. Seth AK, Rawal P, Bagga R, Jain P. Successful colonoscopic fecal microbiota transplantation for active ulcerative colitis: first report from India. Indian J Gastroenterol 2016;35:393-395.

53. Subramanyam C, Pratap N, Gandham P, et al. Influence of probiotic administration on the fecal microbiota in diarrhea-predominant irritable bowel syndrome. Indian J Gastroenterol 2015;34:275-276.

54. Vaishnavi C. Fecal microbiota transplantation for management of Clostridium difficile infection. Indian J Gastroenterol 2014;33:301-307. 